\title{
Up to 11 years of experience with the Melody valved stent in the right ventricular outflow tract
}

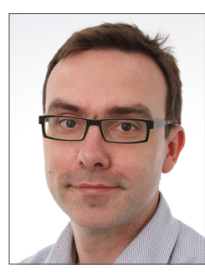

Bjorn Cools ${ }^{1,2}, \mathrm{MD}$; Steven Brown ${ }^{1,2,3}, \mathrm{MD}$, PhD; Werner Budts ${ }^{2,4}, \mathrm{MD}, \mathrm{PhD}$; Ruth Heying ${ }^{1,2}, \mathrm{MD}, \mathrm{PhD}$; Els Troost ${ }^{2,4}, \mathrm{MD}$; Derize Boshoff ${ }^{1,2}, \mathrm{MD}$, PhD;

Benedicte Eyskens ${ }^{1,2}, \mathrm{MD}, \mathrm{PhD}$; Marc Gewillig ${ }^{1,2 *}, \mathrm{MD}, \mathrm{PhD}$

1. Division of Pediatric and Congenital Cardiology, University Hospitals Leuven, Leuven, Belgium; 2. Department of Cardiovascular Sciences, Catholic University Leuven, Leuven, Belgium; 3. Division of Pediatric and Congenital Cardiology, University of the Free State, Bloemfontein, South Africa; 4. Division of Adult Congenital Cardiology, University Hospitals Leuven, Leuven, Belgium

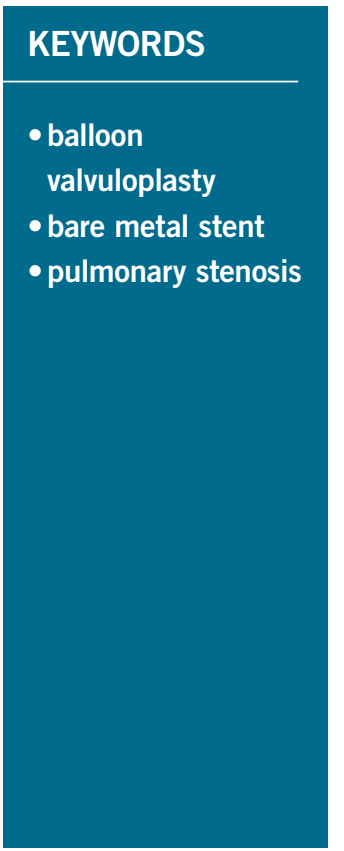

\begin{abstract}
Aims: The aim of the study was to report up to 11 years of follow-up after Melody valve implantation in the pulmonary position.

Methods and results: This was a single-institution non-randomised prospective observational study of all Melody valves in the pulmonary position after discharge between 2006 and $2017(\mathrm{n}=188)$. Mean age was 19.4 years (SD 13.2). The indication was stenosis (45\%), regurgitation (33\%) and mixed (22\%). Prestenting was performed in all except the initial four patients. In stenotic lesions the peak gradient was $36 \mathrm{mmHg}$ PIG (SD 12.0) after 11 years and in regurgitant lesions the maximal regurgitation was $2 / 4$. Stent fractures were observed in $8.6 \%$; only one grade III fracture required redo PPVI. Surgical removal was carried out in seven (3.7\%), redo PPVI in five (2.7\%). Endocarditis was diagnosed in $19(10.2 \%)$ patients at a median of 2.3 years $(0.7-8.8)$ after Melody implantation. Three were surgically removed early because of persistent infection, 16 were sterilised; six required replacement (three surgical, three redo PPVI). There were no valve- or procedure-related deaths.
\end{abstract}

Conclusions: The Melody valve shows a good preserved leaflet function up to 11 years after implantation. The main reason for graft failure was endocarditis, although in half of those patients no reintervention was needed. After pre-stenting, stent fractures led very exceptionally to reintervention.

\footnotetext{
*Corresponding author: Department of Pediatric and Congenital Cardiology, University Hospitals Leuven, Herestraat 49, B-3000 Leuven, Belgium.E-mail: Marc.gewillig@uzleuven.be
} 


\section{Abbreviations}

ICE intravascular cardiac echography

PPVI percutaneous pulmonary valve implantation

PR pulmonary regurgitation

PS pulmonary stenosis

RV right ventricle

RVOT right ventricular outflow tract

\section{Introduction}

Since the first human implantation performed in the year 2000, percutaneous pulmonary valve implantation (PPVI) has established itself as technically feasible with excellent short-term to midterm outcomes over all age groups ${ }^{1-5}$. PPVI results in improvement in right ventricular (RV) function and dimensions, reduction of tricuspid regurgitation, improved electrical stability, exercise ability and a better quality of life ${ }^{6}$. Early adverse events included coronary compression, rupture of the right ventricular outflow tract (RVOT), aortic valve compression, aortic erosion, valve embolisation and tricuspid valve damage. These mostly occurred during initial experiences and have largely been curtailed by better quality assessment practices and technical improvements. Stent recompression, stent fracture and endocarditis have emerged as potential problems during long-term follow-up ${ }^{1,2}$.

The indications for PPVI have changed over time. Initially, the stented valve was developed to prolong conduit function in order to delay surgical valve replacement; it can now be offered for almost any dysfunctional RVOT, even for those without a previous conduit ${ }^{7}$. Extensive medium and long-term data on the performance of the Melody ${ }^{\circledR}$ valved stent (Medtronic, Minneapolis, MN, USA) in the RVOT are not yet available. Follow-up data are required to compare PPVI with existing surgical techniques, and to determine its role when applied to symptomatic patients or even when applied for prognostic reasons ${ }^{8}$.

This aim of this study was to analyse up to 11-year follow-up data of Melody valve implantations in our centre.

Editorial, see page 971

\section{Patients and methods}

This is a single-institution non-randomised prospective observational study. All patients discharged with a Melody valve in the pulmonary position were entered into a registry starting in 2006 with a specified protocol for clinical follow-up. For the purpose of this analysis, the database was closed in December 2017 and included 185 patients.

Standard techniques for implantation were used, as previously described $^{7,9}$. In short, procedures were performed under general anaesthesia and standard prophylactic antibiotic regimens followed. After discharge, endocarditis prophylaxis was recommended to all; no antiplatelet medication was prescribed except when indicated for other reasons.

\section{FOLLOW-UP}

The protocol consisted of follow-up at 1, 3, 6 and 12 months, followed by annual visits thereafter. This included radiography to assess for the presence of stent fractures as well as routine transthoracic echocardiographic examination. Stent fractures were classified as proposed by Nordmeyer et $\mathrm{al}^{10}$. Pulmonary regurgitation (PR) was classified according to a scale of 0 to 4 (none to severe), based mainly on the magnitude of the regurgitation jet on colour Doppler, early termination of diastolic flow as well as right heart dilation.

Endocarditis was diagnosed using the modified Duke criteria. Imaging was carried out by transthoracic echocardiography and, in case of inconclusive images, transoesophageal echocardiography or especially intravascular ultrasound (ICE) was performed ${ }^{11}$.

\section{ETHICS AND STATISTICS}

Assent and informed consent was obtained from patients, their parents or legal guardians. Approval for the study was granted by the local ethics committee (S60373); for inclusions after 2010, adherence to the study protocol was imposed by Belgian medical insurance in order to obtain reimbursement. SPSS, Version 20 (IBM Corp., Armonk, NY, USA) was used for data analysis. Continuous data are expressed as mean and standard deviation or median and range. Paired data were analysed by a paired t-test and the Kruskal-Wallis test. Kaplan-Meier plots were used for survival analyses. A p-value $<0.05$ indicated statistical significance.

\section{Results}

One hundred and eighty-eight $(n=188)$ Melody valves were implanted in 186 patients over this 11-year period. In one patient, a Melody valve was explanted immediately after post-implant dilatation because of coronary compression; this implantation failure was not included in this study. This study therefore reports on follow-up of 188 valves in 185 patients discharged with a Melody valve. Age and weight at implantation can be viewed in Table 1. Two thirds of the patients were male (125 vs. 62 female) and the majority (119/185) were younger than 18 years at implantation.

Table 1. Demographic data. Indication and primary lesion.

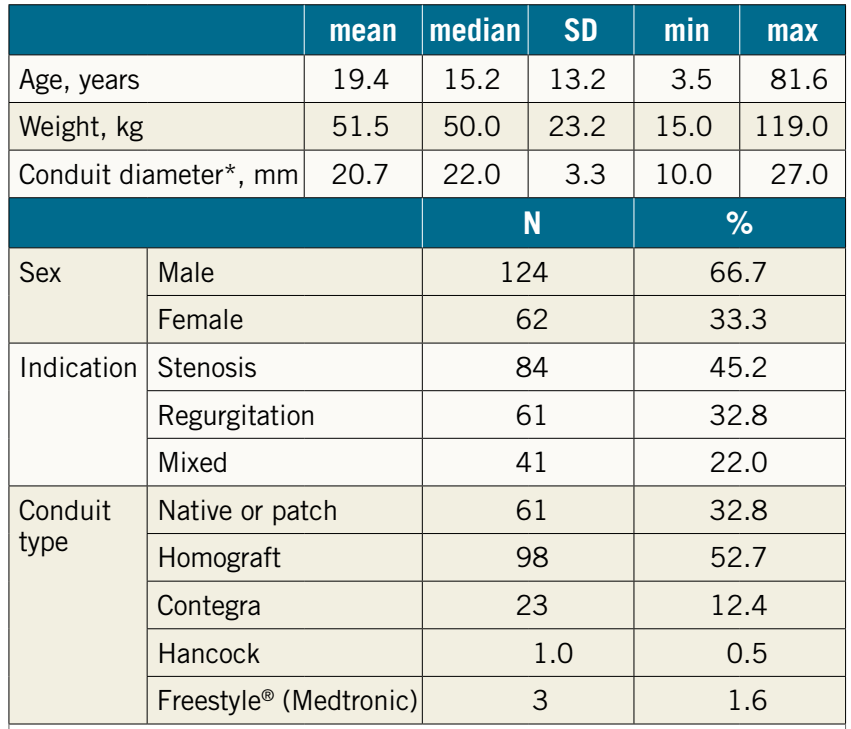

${ }^{*}$ Conduit diameter prior to implant. SD: standard deviation 
The main indications for PPVI were a stenotic RVOT (45\%) followed by dominant PR (33\%) with the remainder being mixed pulmonary stenosis (PS)-PR (22\%). Diagnosis of underlying conditions included tetralogy of Fallot $(n=101) 54.3 \%$, post Ross operation $(n=33) 17.7 \%$, post Rastelli repair $(n=17) 9.2 \%$, pulmonary stenosis $(n=23) 12.4 \%$ and truncus arteriosus $(n=12) 6.5 \%$. The stented valves were implanted in homografts $(52.7 \%)$, in native or patched RVOTs (32.8\%), and in bioprostheses (14.5\%) (e.g., Contegra $^{\circledR}$, Hancock ${ }^{\circledR}$ [both Medtronic], etc.) (Table 1).

Pre-stenting was not performed in the initial four patients (standard recommendations at that stage, early experience). Thereafter and with increasing understanding, RVOT pre-stenting was actively performed and evolved significantly. Stents were implanted to ensure a stable landing zone with no recoil or distortion; care was taken to abolish all gradients prior to Melody valve implantation. Initially, the outflow tract was dilated up to the nominal value of the surgical conduit (standard recommendation at that stage). In the later years, conduits were expanded with covered stents up to 22-24 mm provided that the size of the RVOT and coronary anatomy were acceptable. A total of 226 pre-stents were implanted - one only in 147 patients, two in 26, three in 6 and four stents in 2 patients. Hybrid open-cell bare metal stents were implanted in the conduit-free outflow tracts as described in a previous publication ${ }^{1}$. Various types of stent were used for pre-stenting (CP Stent ${ }^{\mathrm{TM}} \mathrm{n}=161$, covered CP Stent ${ }^{\mathrm{TM}}$ [both NuMED, Hopkinton, NY, USA] $n=31$, AndraStent ${ }^{\mathbb{R}}$ [AndraTec $\mathrm{GmbH}$, Koblenz, Germany] $\mathrm{n}=8, \mathrm{Max}^{\mathrm{TM}}$ LD [Covidien, now Medtronic, Minneapolis, MN, USA] $n=2$, various $n=3$ ). Melody valves were delivered on $18 \mathrm{~mm}(\mathrm{n}=11), 20 \mathrm{~mm}(\mathrm{n}=34)$ and the vast majority on a $22 \mathrm{~mm}(\mathrm{n}=145)$ Ensemble $^{\mathbb{R}}$ system (Medtronic). Of the latter, one was overexpanded to $24 \mathrm{~mm}$ inner diameter.

\section{FOLLOW-UP}

Patients have been followed up for a total of 836 patient years over this 11-year period. At closure of the database in December 2017, clinical and echocardiographic follow-up was complete in all patients, thus with last annual visit later than December 2016 except when censored (death, explant, re-stent, redo PPVI).

\section{GRAFT SURVIVAL}

The overall graft survival was $78 \%$ at 10 years of followup (Figure 1). Thirteen patients $(6.9 \%)$ had failure of the graft requiring replacement: in seven patients the stent was surgically removed due to endocarditis ( $n=6$ : none acute, three early because of ongoing infection, three elective after sterilisation) and subpulmonary stenosis $(n=1)$. In five patients a redo PPVI was performed for stenosis after endocarditis $(n=3)$, in one due to conduit stenosis 10.3 years after initial implantation and in one patient due to a type III stent fracture at 5.2 years after implantation. Redo PPVI was carried out in both with a new Melody valved stent.

\section{PATIENT SURVIVAL}

There were three late deaths, none of which was related to the procedure or related to the valve overall: one patient died from kidney

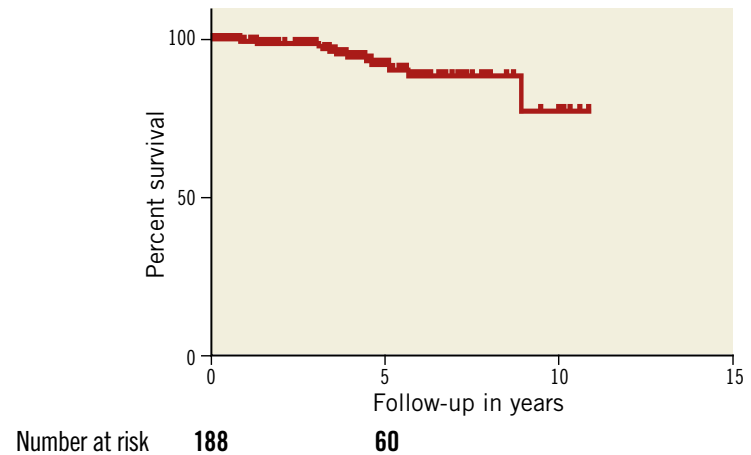

Figure 1. Kaplan-Meier estimated 11-year Melody overall graft survival in RVOT. Graft failure is defined by surgical removal of the Melody valve or redo PPVI with another valved stent.

failure at the age of 85 years, one due to status epilepticus at the age of 20 years and one sudden death during sleep at the age of 26 years (no autopsy was performed).

\section{HAEMODYNAMIC OUTCOMES}

When stenosis was the main indication for PPVI, we observed initially a sharp decline of the peak instantaneous Doppler gradient (PIG) to $23.4 \mathrm{mmHg}$ (SD 11.1), which remained fairly unchanged at $36 \pm 12 \mathrm{mmHg}$ over the RVOT during follow-up (Figure 2). When PR was the dominant lesion, it followed a similar pattern and dropped from median 4/4 to median 0/4 after PPVI (range 0-2) and slightly increased to median $2 / 4$ at 11 years (Figure 3 ). Functional status remained constant after 11 years with the majority of the patients in NYHA functional Class I-II. One patient developed an increased gradient across the valve without any sign of endocarditis: the wall

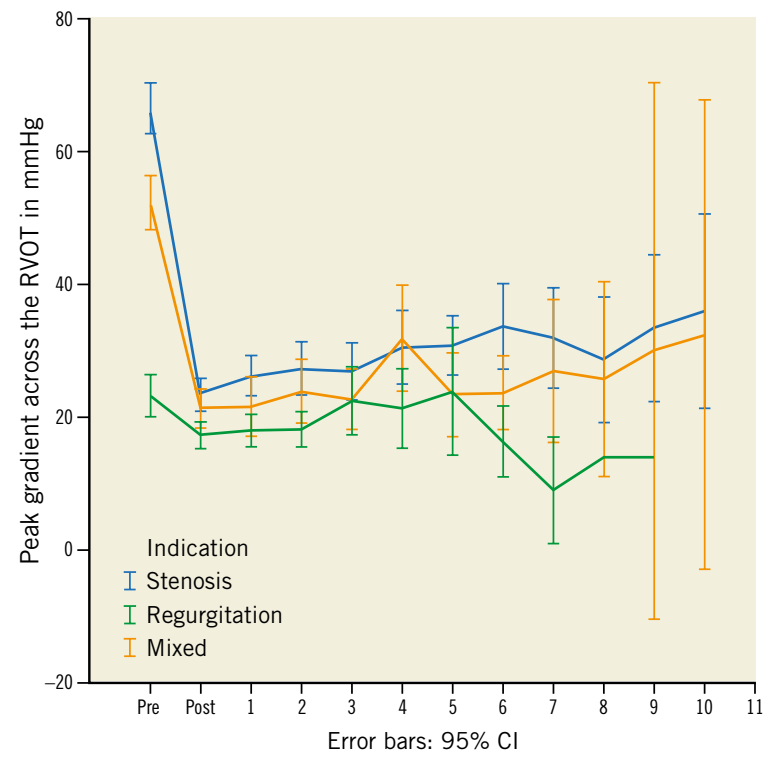

Figure 2. Graft function. Evolution of RVOT peak Doppler gradient $=4(V \max )^{2}$. Depicted are three groups with predominant stenosis (blue), predominant regurgitation (green) and mixed (orange). 


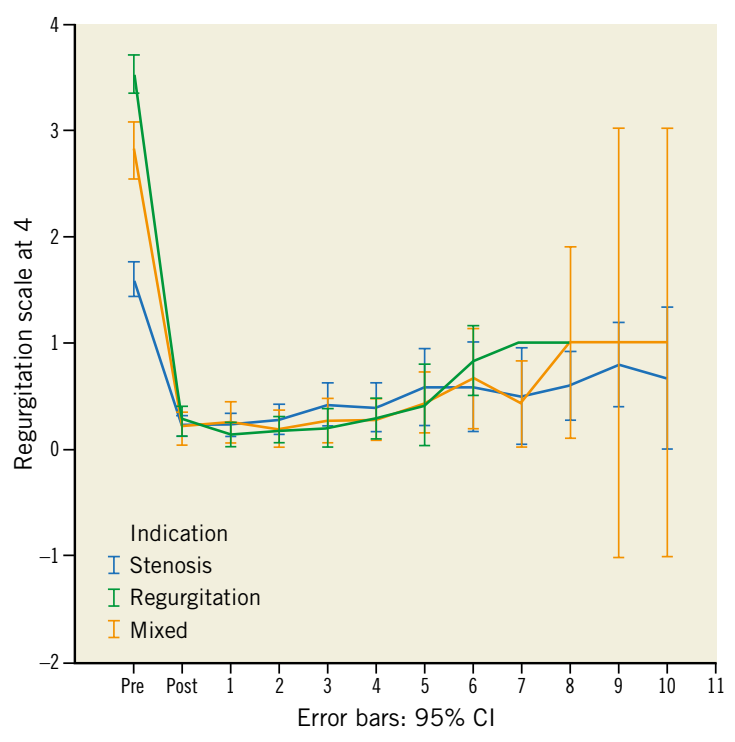

Figure 3. Graft function. Evolution of pulmonary regurgitation (PR). Depicted are three groups with predominant stenosis (blue), predominant regurgitation (green) and mixed (orange).

of the valve was thickened on intravascular ultrasound and angiogram. We could not determine whether this was due to thickening of the conduit wall or a hammock effect of the surrounding tissue. As balloon dilation was unsuccessful due to significant recoil, a bare metal stent was implanted for adequate relief of the gradient, followed four months later by repeat PPVI.

\section{STENT FRACTURES}

Chest radiography was performed on a regular basis (once a year) in $87 \%$ of patients. Stent fractures were observed in 16 patients (8.6\%). In two patients a type III fracture of a covered CP Stent was observed five and seven years after implantation; one of these required redo stenting and redo PPVI because of moderate stenosis. When reviewing the implantation procedure in this case, the pre-stent still showed motion and should have been fortified with an additional stent prior to Melody implantation. In the remaining 14 patients, only type I stent fractures were observed a mean of 4.2 years (SD 2.6) after implant. The relative incidence of stent fracture was $1 / 4(25 \%)$ in the initial four patients who did not receive a pre-stent versus $13 / 182(7.1 \%)$ in the pre-stented group. None of the minor fractures resulted in haemodynamic consequences. Freedom from stent fractures was $82 \%$ at 11 years (Figure 4).

\section{REINTERVENTIONS}

In $21(11.3 \%)$ patients a reintervention was performed during follow-up. Balloon angioplasty to accommodate somatic growth was performed in eight $(4.3 \%)$ patients a mean of 3.4 (SD 1.9) years after implantation. Seven (4.3\%) Melody valves were explanted, six for endocarditis and one electively as a result of progressive subvalvular pulmonary stenosis. Repeat PPVI was required in three patients after curing the endocarditis, in one patient following a type III fracture and in one after "wall thickening".

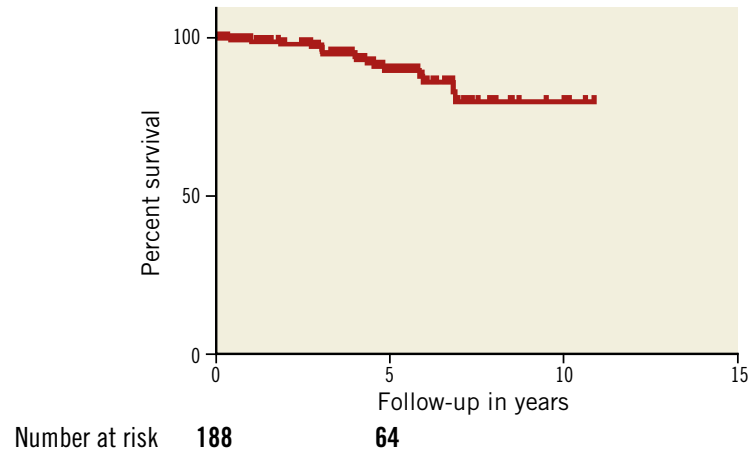

Figure 4. Kaplan-Meier analysis. Freedom from stent fractures (study period 2006-2017).

\section{ENDOCARDITIS}

Endocarditis was diagnosed in $19(10.2 \%)$ patients at a median time of 2.3 years (range $0.7-8.9$ ) after Melody implantation. The median age at the time of endocarditis was 16.2 years (range: 8.0-45.6) with a strong male preponderance $(16 / 19,84.2 \%)$. Freedom from endocarditis was $84.9 \%$ and $76.2 \%$ after five and 10 years, respectively (Figure 5). The original substrate in which the Melody valve was implanted in these cases was homograft $(n=10)(52.6 \%)$, native or patched RVOT $(n=6)(31.6 \%)$ and Contegra type valve grafts $(n=3)(15.8 \%)$. Peak instantaneous gradients over the RVOT in patients presenting with endocarditis had increased significantly, from a median of $23 \mathrm{mmHg}$ (range: $10-42)$ to a median of $65 \mathrm{mmHg}$ (range: 18-110) $(\mathrm{p}=0.001)$. Positive bacterial identification was obtained in $89.4 \%$ of the cases: Staphylococci species $(n=6)$, Streptococcus viridans $(n=5)$, HACEK group $(n=2)$ and other $(n=4)$. In two patients no organisms were cultured. A probable entry point could be determined in $15 / 19$ patients $(78.9 \%)$, predominantly dental and in some from the skin. Three were surgically removed early $(4,17$ and 25 days) because of persistent infection; 16 were sterilised after four to six weeks of antibiotics; six required eventual replacement because of obstruction (three surgical, three redo PPVI with a SAPIEN valve [Edwards Lifesciences, Irvine, CA, USA]). In the latter patients we first waited a minimum of six weeks

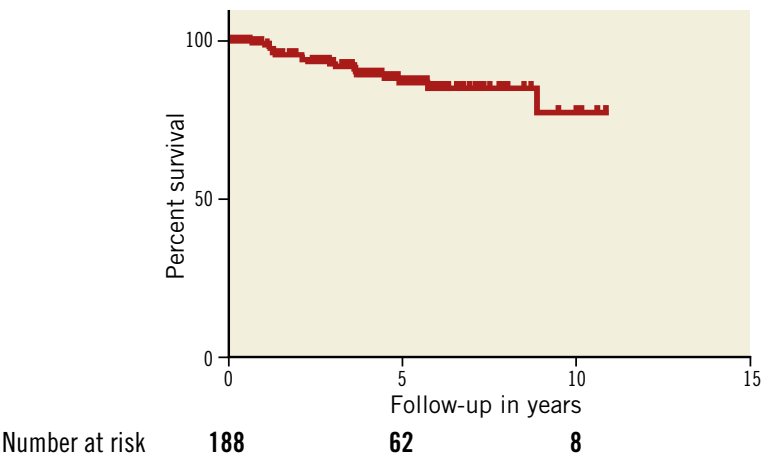

Figure 5. Kaplan-Meier analysis. Freedom from endocarditis (study period 2006-2017). 
before pre-stenting, and again a minimum of six weeks prior to redo PPVI to ascertain eradication of the infection.

\section{Discussion}

As time progresses and experience increases, PPVI is establishing itself as a very competitive option for the treatment of RVOT dysfunction. The most important finding of this interim analysis is that the Melody valve exhibited good graft survival with good function up to 11 years of follow-up for patients who remained free from endocarditis. Gradients across the RVOT did not change significantly and pulmonary regurgitation remained low. Stent fractures with haemodynamic consequences are exceptional after adequate pre-stenting, but endocarditis remains a challenge.

\section{MELODY VALVE FUNCTION}

Overall graft survival was $78 \%$ after 10 years of follow-up; the main reason for graft failure was valve dysfunction due to endocarditis $(10 / 13 ; 77 \%)$. If patients remained free from endocarditis, there was little progression of obstruction and/or regurgitation. This can be attributed to a number of factors. The procedural protocol includes adequate pre-stenting of the RVOT with the aim of ensuring mechanical stability of the landing zone, underscored by the number of stents used during pre-stenting. The aim of this protocol is to ensure strength (resistance to deformity) of the scaffolding with redistribution of stresses over the coupled stent system resulting in improved stented valve function. Even though disadvantages such as fretting, leverage and galvanic corrosion may occur, the success of this approach is illustrated by the fact that none of our patients presented with acute collapse of the stented valve and only a few demonstrated signs of mild recompression ${ }^{12}$.

The minimal stenosis and regurgitation indicate preserved good function of the graft leaflets over the period of our analysis. As opposed to surgical conduits, where obstruction frequently occurs at the anastomosis of "normal" tissue to conduits, the joined stent scaffolding in the landing zone potentially prevents initial direct contact of the graft tissue with that of the patient at the proximal and distal ends of the landing zone. Furthermore, disturbed patterns of flow are more likely with surgical implants not only due to limitations at implantation, but also due to compression, kinking, shrinkage and fibrosis over time. Adequate pre-stenting aims to prevent external compression and streamlines flow as a result of straightening of distorted conduits and by achieving a nice circular shape - all of which are likely to improve valve function further. Pre-stenting is thus important for a number of reasons as the omission of pre-stenting during the initial London and US experiences revealed ${ }^{2}$. Similar to surgical experiences, where larger conduits provide better longevity of a valve, it would be logical to ensure an adequate diameter of the RVOT during pre-stenting. We currently aim for a 22 to $24 \mathrm{~mm}$ landing zone, if permitted by the anatomy and coronary proximity. In the majority of our patients (75.3\%), valves were implanted using the largest $(22 \mathrm{~mm})$ delivery system and expanded up to $22 \mathrm{~mm}$ inner diameter.

\section{REINTERVENTIONS}

Reinterventions were performed in $11.3 \%$ of patients. These findings are in agreement with the medium-term results of the US trial where the freedom from reintervention after five years was $91 \%{ }^{13}$. The main reason for surgical explantation (6/7) of the graft or percutaneous redo PPVI (3/5) was endocarditis. One valve was overstented because of a grade III pre-stent fracture. One valve was electively explanted 3.5 years after implantation due to progressive subpulmonary obstruction; the Melody valve itself still had normal function and appearance, with an endothelial covering of the valve leaflets. In this patient, the initial residual infundibular gradient was mild, but continued to increase as the right ventricle size regressed as a result of reduced volume loading after Melody implantation. Cognisance of this occurrence should be taken, since right ventricular remodelling after PPVI may amplify gradients proximal to the landing zone. Balloon angioplasty to accommodate for somatic growth is a decided advantage of a percutaneous valve and adds to the attraction of PPVI. We considered this as an elective treatment and not as a forced reintervention.

\section{STENT FRACTURES}

Fractures of the stented RVOT (7.5\%) in our series were lower than in most reports ${ }^{2,10,14}$. The use of multiple overlapping stents and the use of simple X-ray (frontal and lateral projection) may have precluded the detection of small fractures of the Melody stent; however, Doppler echocardiography excluded a clinically relevant change of valve function, which is the final goal of this treatment. Fractures were mostly of class I with no haemodynamic compromise and we observed only two grade III fractures of a prestent. In one patient, a redo PPVI was performed because the clinician observed mild deformation of the conduit in the presence of a moderate Doppler gradient of $45 \mathrm{mmHg}$; prophylactically an additional outflow tract stent and Melody valve were placed. An explanation for the observed low frequency of stent fractures and extremely low occurrence of haemodynamically important fractures can possibly be found in the rigid pre-stenting protocol: aggressive pre-stenting until no more motion of the "tube" is observed. We hypothesise that this strategy decreases strain amplitude and metal fatigue, thereby delaying or even avoiding fracture during a human lifetime. This is supported by previous analysis of mechanical stress bench testing of stents ${ }^{12}$.

\section{ENDOCARDITIS}

Endocarditis is an important threat for any bioprosthetic valve, including the Melody valve. Several series have reported incidence rates of endocarditis of between 7.5 and $16 \%$ in bovine valves $^{15}$. In our series endocarditis occurred in $10.2 \%$ of the implants. In $10 / 19(52.6 \%)$ the endocarditis was cured with antibiotics only, without significant change in valve function. No deaths occurred and no urgent surgery was required since all patients, carers and general practitioners were instructed to monitor for early signs of endocarditis; additionally, there is a short and fast track for referral to the implant centre in case 
of suspicion of endocarditis. Of note is that a "sudden" significantly increased gradient over the RVOT should alert the consulting cardiologist to the possible presence of endocarditis.

The study was not powered to identify specific factors, but there is a strong male preponderance and mostly young adolescents appear to be at higher risk. Youngsters in the pubertal age group are difficult to control in terms of hygienic measures (dental/skin). In $15 / 19$ patients a clear entry point for endocarditis could be identified. Following extensive education of patients, their parents and carers, not allowing overruling or deviation from the ESC guidelines, we have observed a reduction of endocarditis from a maximal annual incidence of $2.6 \%$ down to less than $1.0 \%$ currently. All patients were advised to follow a rigorous schedule of dental and skin hygiene and to take adequate prophylaxis prior to highrisk procedures. Similar information was conveyed to their local practitioners and dentists. It should be noted that the 2015 ESC guidelines specify transcatheter heart valves to be at higher risk (IIa evidence) whilst the 2007 AHA guidelines only allude to prosthetic valves ${ }^{16,17}$.

As a result, this has been confusing for practitioners and dentists and, as far as our country is concerned, it has led to a more liberal attitude concerning subacute bacterial endocarditis (SBE) prophylaxis. In view of the findings of this study and other reports, it seems prudent to promote that biological percutaneous valves should adhere to strict antibiotic prophylactic measures.

The current strategy in our centre is not to give antiplatelet therapy. The use of antiplatelet therapy in preventing microthrombi and possible prevention of infective endocarditis (IE) is being studied $^{18-21}$. However, there is currently no evidence that antiplatelet therapy with aspirin only reduces endocarditis in this situation; with our current strategy we have reduced the annual incidence of endocarditis to below $1.0 \%$ year, which is lower than similar cohorts with aspirin. The jury is still out on this question!

\section{COMPARISON TO SURGERY}

A recent contemporary multicentre surgical trial of decellularised $(n=163)$ and standard allografts $(n=124)$ with similar numbers over a comparable period showed that freedom from conduit dysfunction at 10 years was $83 \%$ for decellularised conduits and $58 \%$ for standard allografts $(p<0.001)^{22}$. In a review of best evidence by Abbas et al, freedom from reintervention and graft failure varied from $47 \%-72 \%$ after 10 years for various conduits ${ }^{23}$. Similar findings were reported for Contegra conduits in children where freedom from reoperation was $75 \%$ at five years. Our results show that the Melody valve had a freedom from conduit dysfunction of $78 \%$ after 10 years, indicating that it is highly competitive compared to current conduits and might perform even better if endocarditis were contained. Furthermore, if adaptation for somatic growth is excluded (which is not an option for most surgical conduits), the Melody valve required a reintervention in $7 \%$ of the cases within 11 years of follow-up. This is low in comparison to the $10 \%$ risk of reintervention for the decellularised conduits and even $27 \%$ for standard allografts $22-25$.
Possible reasons for the better performance of the Melody valve as opposed to surgical conduits include absence of retraction or shrinkage, as is frequently observed at the muscular cuff of a homograft or at the distal anastomosis of a Contegra. Furthermore, a stented conduit is more resistant to the external compression typically exerted by the sternum and aorta or internal retraction due to fibrosis of the biological tissue.

\section{Limitations}

The study suffers from the observational design and only reflects practice at one centre. Radiology of stent integrity was based on standard chest views and with multiple pre-stenting minor fractures may not have been observed. However, haemodynamic compromise was easily excluded or confirmed by Doppler echocardiography. Patients were not randomly selected for inclusion and no control group was used, making it a heterogeneous sample. The protocol for pre-stenting evolved during the study. As a result of geographical location, some patients could not follow all the elements of the protocol (annual chest radiography) and some of the follow-ups were performed at local centres.

\section{Conclusions}

The Melody valve shows good graft survival up to 11 years. Gradient relief can be attributed to pre-stenting, with a lasting result over the study period. Leaflet function remained good: no significant stenosis or regurgitation. Endocarditis remains an important issue, which might be reduced by stringent adherence to prophylactic measurements. In terms of longevity, PPVI with the Melody valved stent compares favourably with surgical grafts.

\section{Impact on daily practice}

The Melody valved stent shows a good preserved function for the current length of follow-up. Endocarditis is the main reason for graft failure in this series.

\section{Funding}

This work was supported by grants from the Eddy Merckx Research Foundation and the Belgian Foundation for Research in Pediatric Cardiology (FNRCP).

\section{Conflict of interest statement}

M. Gewillig is a proctor for NuMED, Medtronic and Edwards. S. Brown is a proctor for Medtronic. The other authors have no conflicts of interest to declare.

\section{References}

1. Cools B, Budts W, Heying R, Boshoff D, Eyskens B, Frerich S, Troost E, Gewillig M. Medium term follow-up after percutaneous pulmonary valve replacement with the Melody ${ }^{\circledR}$ valve. Int $J$ Cardiol Heart Vasc. 2015;7:92-7.

2. Cheatham JP, Hellenbrand WE, Zahn EM, Jones TK, Berman DP, Vincent JA, McElhinney DB. Clinical and hemody- 
namic outcomes up to 7 years after transcatheter pulmonary valve replacement in the US melody valve investigational device exemption trial. Circulation. 2015;131:1960-70.

3. Borik S, Crean A, Horlick E, Osten M, Lee KJ, Chaturvedi R, Friedberg MK, McCrindle BW, Manlhiot C, Benson L. Percutaneous pulmonary valve implantation: 5 years of follow-up: does age influence outcomes? Circ Cardiovasc Interv. 2015;8:e01745.

4. Fraisse A, Aldebert P, Malekzadeh-Milani S, Thambo JB, Piechaud JF, Aucoururier P, Chatelier G, Bonnet D, Iserin L, Bonello B, Assaidi A, Kammache I, Boudjemline Y. Melody ${ }^{\circledR}$ transcatheter pulmonary valve implantation: results from a French registry. Arch Cardiovasc Dis. 2014;107:607-14.

5. Armstrong AK, Balzer DT, Cabalka AK, Gray RG, Javois AJ, Moore JW, Rome JJ, Turner DR, Zellers TM, Kreutzer J. One-year follow-up of the Melody transcatheter pulmonary valve multicenter post-approval study. JACC Cardiovasc Interv. 2014;7:1254-62.

6. Nguyen HH, Shahanavaz S, Van Hare GF, Balzer DT, Nicolas R, Avari Silva JN. Percutaneous pulmonary valve implantation alters electrophysiologic substrate. J Am Heart Assoc. 2016 Sep 30;5(10).

7. Cools B, Brown SC, Heying R, Jansen K, Boshoff DE, Budts W, Gewillig M. Percutaneous pulmonary valve implantation for free pulmonary regurgitation following conduit-free surgery of the right ventricular outflow tract. Int J Cardiol. 2015;186: 129-35.

8. Pagourelias ED, Daraban AM, Mada RO, Duchenne J, Mirea O, Cools B, Heying R, Boshoff D, Bogaert J, Budts W, Gewillig M, Voigt JU. Right ventricular remodelling after transcatheter pulmonary valve implantation. Catheter Cardiovasc Interv. 2017;90:407-17.

9. Boshoff DE, Cools BL, Heying R, Troost E, Kefer J, Budts W, Gewillig M. Off-label use of percutaneous pulmonary valved stents in the right ventricular outflow tract: time to rewrite the label? Catheter Cardiovasc Interv. 2013;81:987-95.

10. Nordmeyer J, Khambadkone S, Coats L, Schievano S, Lurz P, Parenzan G, Taylor AM, Lock JE, Bonhoeffer P. Risk stratification, systematic classification, and anticipatory management strategies for stent fracture after percutaneous pulmonary valve implantation. Circulation. 2007;115:1392-7.

11. Li JS, Sexton DJ, Mick N, Nettles R, Fowler VG Jr, Ryan T, Bashore T, Corey GR. Proposed modifications to the Duke criteria for the diagnosis of infective endocarditis. Clin Infect Dis. 2000;30:633-8.

12. Cools B, Brown S, Wevers M, Humbeeck JV, Boshoff D, Verdonckt C, Gewillig M. Right ventricle outflow tract prestenting: In vitro testing of rigidity and corrosion properties. Catheter Cardiovasc Interv. 2018;91:285-91.

13. McElhinney DB, Hellenbrand WE, Zahn EM, Jones TK, Cheatham JP, Lock JE, Vincent JA. Short- and medium-term outcomes after transcatheter pulmonary valve placement in the expanded multicenter US melody valve trial. Circulation. 2010;122: 507-16.
14. Nordmeyer J, Coats L, Lurz P, Lee TY, Derrick G, Rees P, Cullen S, Taylor AM, Khambadkone S, Bonhoeffer P. Percutaneous pulmonary valve-in-valve implantation: a successful treatment concept for early device failure. Eur Heart J. 2008;29:810-5.

15. O’Donnell C, Holloway R, Tilton E, Stirling J, Finucane K, Wilson N. Infective endocarditis following Melody valve implantation: comparison with a surgical cohort. Cardiol Young. 2017;27: 294-301.

16. Van Dijck I, Budts W, Cools B, Eyskens B, Boshoff DE, Heying R, Frerich S, Vanagt WY, Troost E, Gewillig M. Infective endocarditis of a transcatheter pulmonary valve in comparison with surgical implants. Heart. 2015;101:788-93.

17. Habib G, Lancellotti $P$, Antunes MJ, Bongiorni MG, Casalta JP, Del Zotti F, Dulgheru R, El Khoury G, Erba PA, Iung B, Miro JM, Mulder BJ, Plonska-Gosciniak E, Price S, RoosHesselink J, Snygg-Martin U, Thuny F, Tornos Mas P, Vilacosta I, Zamorano JL; ESC Scientific Document Group. 2015 ESC Guidelines for the management of infective endocarditis: The Task Force for the Management of Infective Endocarditis of the European Society of Cardiology (ESC). Endorsed by: European Association for Cardio-Thoracic Surgery (EACTS), the European Association of Nuclear Medicine (EANM). Eur Heart J. 2015;36:3075-128. 18. Eisen A, Shapira Y, Sagie A, Kornowski R. Infective endocarditis in the transcatheter aortic valve replacement era: comprehensive review of a rare complication. Clin Cardiol. 2012;35:E1-5.

19. Jung CJ, Yeh CY, Shun CT, Hsu RB, Cheng HW, Lin CS, Chia JS. Platelets enhance biofilm formation and resistance of endocarditis-inducing streptococci on the injured heart valve. J Infect Dis. 2012;205:1066-75.

20. Hoen B. Platelets and Platelet Inhibitors in Infective Endocarditis. Curr Infect Dis Rep. 2002;4:299-303.

21. Malekzadeh-Milani S, Ladouceur M, Patel M, Boughenou FM, Iserin L, Bonnet D, Boudjemline Y. Incidence and predictors of Melody ${ }^{\circledR}$ valve endocarditis: a prospective study. Arch Cardiovasc Dis. 2015;108:97-106.

22. Bibevski S, Ruzmetov M, Fortuna RS, Turrentine MW, Brown JW, Ohye RG. Performance of SynerGraft Decellularized Pulmonary Allografts Compared With Standard Cryopreserved Allografts: Results From Multiinstitutional Data. Ann Thorac Surg. 2017;103:869-74.

23. Abbas JR, Hoschtitzky JA. Which is the best tissue valve used in the pulmonary position, late after previous repair of tetralogy of Fallot? Interact Cardiovasc Thorac Surg. 2013;17:854-60.

24. Pardo Gonzalez L, Ruiz Ortiz M, Delgado M, Mesa D, Villalba R, Rodriguez S, Hidalgo FJ, Alados P, Casares J, Suarez de Lezo J. Pulmonary homograft stenosis in the Ross procedure: Incidence, clinical impact and predictors in long-term follow-up. Arch Cardiovasc Dis. 2017;110:214-22.

25. Yong MS, Yim D, d'Udekem Y, Brizard CP, Robertson T, Galati JC, Konstantinov IE. Medium-term outcomes of bovine jugular vein graft and homograft conduits in children. ANZ J Surg. 2015;85:381-5. 